\title{
La función y alcances de la Teoría de los Tres Mundos en la filosofía popperiana
}

\section{Function and scope of the Three Worlds Theory in Popper's philosophy}

\section{A função e alcances da Teoria dos Três Mundos na filosofia popperiana}

Iván Briceño Ríos ${ }^{1}$

Recibido: 31/07/2015 - Aceptado: 21/09/2015

\begin{abstract}
Resumen
El artículo intenta proponer una alternativa de comprensión de una de las propuesta popperianas que más ha dado que hablar desde la segunda mitad del siglo XX, como es su Teoría de los Tres Mundos. Este camino interpretativo contempla comprender esta teoría desde el lugar que ésta tiene en la filosofía popperiana, como así también desde sus alcances filosóficos, los cuales son de carácter cosmológico y cognitivista pluralista. Para finalmente, proponer que la Teoría de los Tres Mundos representa el carácter fundamental de la filosofía popperiana en términos de su Racionalismo Crítico. En el que la filosofía popperiana se considera libre para discutir y cambiar de plan si el tema lo justifica.
\end{abstract}

Palabras clave: Teoría de los Tres Mundo - filosofía popperiana - cosmología cognitiva - racionalismo crítico.

\begin{abstract}
The article is aimed to propose an alternative for understanding one of the most controversial Popperian proposals from the second half of the Twentieth Century, as is his Theory of the Three Worlds. For this interpretive trail it is necessary to understand this theory from its place in Popper's philosophy, as well as from its philosophical scopes, which are
\end{abstract}

1 Chileno. Licenciado en Filosofía de la Universidad Alberto Hurtado de Chile. Magíster en Filosofía de la Pontificia Universidad Católica de Chile. Doctor (c) en Filosofía de la Pontificia Universidad Católica de Chile. Actualmente es profesor de planta de la Universidad Católica Silva Henríquez. Contacto: ibriceno@ucsh.cl 
cosmological and pluralistic cognitive. The article finally proposes that the Theory of the Three World represents the fundamental character of Popper's philosophy in terms of its critical rationalism where the Popper's philosophy is considered free to discuss and change according to the topic.

Keywords: Theory of the Three Worlds - Popper's philosophy - cognitive cosmology - critical rationalism.

\section{Resumo}

O artigo tenta propor uma alternativa de compreensão de uma das propostas popperianas que mais tem dado de falar a partir da segunda metade do século XX, como é sua Teoria dos Três Mundos. Esta trilha interpretativa contempla compreender esta teoria desde o lugar que esta tem na filosofia de popperiana, assim também desde seus alcances filosóficos. Os quais são de caráter cosmológico e cognitivista pluralista. Para finalmente propor que a Teoria dos Três Mundos representa o caráter fundamental da filosofia de popperiana em termos de seu Racionalismo Crítico. No qual, onde a filosofia de Popper é considerada livre para discutir e mudar de planos, se o tema o justifica.

Palavras-chave: Teoria dos Três Mundos - filosofia popperiana - cosmologia cognitiva - racionalismo crítico.

\section{Introducción}

El pensamiento de Karl Popper es largamente reconocido por sus alcances en el campo epistemológico o respecto de la teoría del conocimiento científico. Sin embargo, en la década del sesenta irrumpe y sorprende en el escenario filosófico con una suerte de giro en su pensamiento. Este viraje, como él lo define, «provocador»y «desafiante» es "una especie de argumento biológico o evolucionista" (Popper 2001b 111), el cual se traduce en lo que será su Teoría de los Tres Mundos (TTM).

Esta teoría popperiana es un tanto compleja, farragosa, metafísica, abstrusa, inusual; es muy difícil de entender, como lo reconoce el propio autor vienés en varios de sus textos. No obstante, su importancia no es menor, ya que en ella se presenta, en primer lugar, un pensamiento maduro. Además, esta propuesta teórica tiene alcances más amplios que los meramente epistemológicos, por ejemplo abriéndose 
a campos como el cosmológico, el evolutivo e incluso el psicológico. $Y$ finalmente, esta teoría tiene un carácter controversial ${ }^{2}$ en la actitud filosófica popperiana, debido a que el pensador extrema en ella su afán teórico, al punto de asirse a un "clavo ardiendo" (Popper 1994b 254).

Como podemos apreciar, estamos frente a una propuesta teórica tardía, extensa en lo que son sus campos de incumbencia y crítica en lo que atañe al filosofar propio del pensador vienés. De ahí que se hace no sólo importante, sino también interesante un estudio respecto de la idea y actitud filosófica que el pensador impregna en ella. Como es el caso en lo que son sus aspectos gnoseológicos, producto de que la TTM, según Karl Popper, representa una "revolución psicológica" (Popper 2001b 149) especialmente en el estudio de la mente 3 .

Para lo anterior proyectamos desarrollar una exposición acerca de la TTM, proponiendo como hipótesis «un optimismo cognitivista de la mente en términos de un oscilar metafísico-cosmológico en lo que respecta a los enigmas del universo, que como dice el propio filósofo, "podríamos darle el nombre de fe irracional en la razón" (Popper 1994a 398)» Para esto último creemos necesario, en primer lugar, exponer algunos de los lineamientos de la filosofía popperiana que permitan vislumbrar la función de la TTM en ella. Para luego

\footnotetext{
2 Una muestra de ese carácter controversial o polémico de este despliegue, en el pensamiento popperiano, es el hecho de que en su obra Poverty of Historicism, al comienzo del apartado ¿Existe una Ley de la Evolución? Leyes y Tendencias, Karl Popper presenta una crítica al evolucionismo, exponiendo: "de acuerdo con el profesor Raven cuando, en su Science Religion, and the Future (1943), Ilama a este conflicto -evolucionista- «una tormenta en una taza de té victoríana»". Sin embargo, el pensador vienés, unos años más tarde, en su obra Objective Knowledge, en el apartado Of Clouds and Clocks reconoce, con "vergüenza [...] que esa taza de té se ha convertido, después de todo, en mi taza de té y con ella he de comerme mi humilde pastel". Como podemos apreciar, esta situación pone en evidencia no sólo un cambio en la idea popperiana respecto del evolucionismo, sino que asimismo hace suyo lo que en 1945 criticó, como son el carácter de ley universal de la naturaleza y el afán holístico del evolucionismo. 3 Basta además hacer un recorrido por las obras popperianas, posteriores a la década del 60 -giro biológico-, para advertir que el tema de lo mental es recurrente, partiendo por varios de los ensayos recogidos en Objective Knowledge (1972), o en su coautoría en The Self and Its Brain (1977) o en Knowledge and the Body-mind Problem. In Defence of Interaction (1994), entre otros.
} 
describir esta teoría como una cosmología en claves cognitivistas. Finalmente levantar el escenario de discusión crítica que la TTM tiene con filosofías de la mente contemporáneas, con el fin de mostrar esos alcances impensados e indefinidos de la propuesta popperiana. Estos tres elementos nos llevarán a abrir un marco de interpretación de la TTM, que se circunscribe más al Racionalismo Crítico, que a la lógica de la investigación científica popperiana. Que responde a un carácter "racionalista -dice Popper- muy especial. No estoy seguro de que mi racionalismo sea aceptable" (Popper 2001b 156).

\section{Desarrollo}

\section{La profundidad del pensamiento popperiano}

Si se revisa lo que ha sido la obra popperiana anterior a la década del sesenta, inmediatamente se cae en lo que son sus alcances epistemológicos. Esto es, forzosamente nos precipitamos a lo que el propio filósofo llama su "teoría del conocimiento científico" (Popper 2001a 108). Esta situación, que delata un cierto velo epistemológico en la obra de Popper de aquella época, lo condena a ser tratado exclusivamente como un filósofo de la ciencia.

Al analizar el arranque de este eclipse, que casí ineludiblemente ostenta la filosofía popperiana, rápidamente caemos en una lógica del descubrimiento científico o en su cercenada primera obra: Logik der Forschung de 1934. En ella se da a grandes rasgos, una investigación teórica respecto de la «lógica del pensamiento» por sobre «psicología de éste». Lo que se traduce en una apuesta por el formalismo antipsicologista, que se concentrará en el estudio de los pasos o formas lógicas que da el conocimiento en su ampliación. Todo ello irá en desmedro de la atención de los hechos psicológicos que asisten en el conocimiento. Esta propuesta asumida por Popper, va a generar que su trabajo sea tempranamente, obnubilado por una "epistemología objetivista" (Popper 1994b 74). Si bien este hecho obtendrá algunos frutos teóricos, en lo referente: «al criterio de demarcación», «al problema de la inducción», «el progreso 
de la ciencia», «el conocimiento científico», «la superación de la metafísica», «la existencia de auténticos problemas filosóficos», «el análisis del lenguaje», entre otros. Sin embargo, todo este producto teórico no permitió divisar el verdadero afán, o para ser más justo, en el sentido que está la base de su filosofía y que será desplegado en toda su vida intelectual. Un escueto decurso de cómo es que se arriba a este traspié nos puede develar aquello que está en sombras en el interés filosófico popperiano.

Si recordamos el Prefacio de la primera edición de la Logik der Forschung de 1934, Popper hace explícito que la labor filosófica se encuentra en una posición muy desigual con respecto al trabajo científico, puesto que la primera "no se enfrenta con una estructura organizada, sino más bien con algo que se asemeja a un montón de ruinas (aunque con un tesoro sepultado debajo)" (Popper 2001a 14). Haciéndonos ver, de forma destacada, un anhelo esperanzador respecto de la filosofía y al mismo tiempo la necesidad de desplegar un estudio epistemológico que pueda reconstruir -desenterrar- la labor de ella. Esto último tiene por fin el hecho de que ésta enfrente y proponga reales problemas filosóficos. Para ello, el pensador vienés arrancará desde dos tesis centrales; estas son, "no hay un método propio de la filosofía" (Popper $2001^{\text {a }} 16$ ) y "el problema central de la epistemología ha sido siempre, y sigue siéndolo, el aumento del conocimiento" (Ibíd.).

Lo primero apunta al hecho de que no hay un típico o exclusivo proceder de la filosofía, y lo segundo es que si queremos dar con una estructura metodológica para ella, sólo será a través del estudio del aumento del conocimiento. No obstante, para el autor vienés, el gran referente para aquella tarea será la labor científica. Pues, "el mejor modo de estudiar el aumento del conocimiento es estudiar el del conocimiento científico" (Ibíd.), debido a que "parece evidente que el aumento de este último -refrenda el autor-es el caso más importante y más interesante del aumento del conocimiento"(Id. 19). En efecto, pese a su interés por la filosofía y por revitalizar su potencial, será esta última idea la que traslade a Karl Popper de lleno a la filosofía de la ciencia, eclipsando su real intención teórica para con los reales problemas filosóficos. 
En el Prefacio de la edición inglesa a la Logik der Forschung de 1958, Popper planteará: "Por mi parte, me interesan la ciencia y la filosofía exclusivamente porque quisiera saber algo del enigma del mundo en que vivimos" (Popper 2001 a 23), exponiéndonos más claramente ese propósito esperanzador respecto de la filosofía de la década del treinta. Así mismo, que el camino a seguir por parte de ésta era el conocimiento del mundo, a ese mundo "que los filósofos llaman un «mundo externo»" (Popper 1994b 27). Evidenciando que su interés, como lo plantea en una conferencia pronunciada en la Aristotelian Society en 1958, es que la filosofía sea cosmología, lo cual se traduce en una investigación respecto del mundo y sus problemas. $Y$ así se lo hace explícito al Profesor Jesús Mosterín, en 1989, cuando éste le pregunta: "Sir Karl, ¿sigue siendo el universo el tema que más le interesa? -Popper responde: como ya dije en mi Lógica de la Investigación Científica, lo que más me interesa es el universo. [...] Sí; sigo estando muy interesado en el universo y en todo lo que ha emergido en él" (Mosterin 1989 10).

Para Popper, la clave para afrontar este estudio cosmológico, es dar cuenta que éste -el mundo- "surge fuera de la filosofía" (Popper 2001 100), es algo externo a la filosofía. De esta forma, el mundo no es un problema o un concepto que lo inventen los filósofos, sino que es algo que asiste frente a ellos de manera problemática. Haciendo que el centro del empeño filosófico popperiano se plasme en el "problema de entender el mundo -incluido nosotros y nuestro conocimiento como parte de él"' (Popper 2001a 16).

Como podemos apreciar, el real interés filosófico popperiano, incluido el epistemológico, está en el mundo y en el carácter enigmático de éste, que exige enfrentarse a él problematizándolo, anteponiendo una "sensibilidad para con los problemas -del mundo-y la ardiente pasión para con ellos" (Popper 2001b 1001), que el autor vienés concibe en términos de una cosmología. En ella se asume como principio metodológico, una suerte de temple en el que somos nosotros quienes conjeturamos las teorías respecto del mundo y somos nosotros quienes las criticamos. No obstante, el mundo no consiste en un «todo» cerrado de sucesos, sino como un conjunto o una multitud de eventos 
problemáticos siempre abiertos o no totalmente previstos. Ante el cual, la filosofía pretende explicarlos - problematizarlos o criticarlos-, como un intento de aprender más acerca de ellos.

Precisamente, asuminedo esta filosofía cosmológica se abre la comprensión de pensamiento popperiano. Huyendo en parte de ese enclaustramiento en el ámbito epistemológico y al mismo tiempo abriéndose a un giro en su filosofía, en términos de que el "mayor enigma de la cosmología [...] es que el universo sea, en cierto sentido creador: que haya creado la vida y, a partir de ella, la mente -nuestra conciencia- que ilumina el universo y que, a su vez, es creadora" (Popper 1980 69).

La filosofía -cosmológica- popperiana se va a desarrollar desde una «dualidad» constituida por los hechos del mundo, que si bien no determinan al filósofo, sí lo desafían críticamente a volcarse sobre ellos, a partir del cual los sucesos del mundo van a ser problematizados o teorizados. La filosofía popperiana tendrá enfrente algunos problemas reales y contextuales del mundo, y por otro una filosofía expresada en su Racionalismo Crítico, con el cual problematiza o enfrenta, eso sí, yendo más allá de un mero proceder o regla metodológica, puesto que este racionalismo será un "credo moral" (Popper 1997 15); "una manera de pensar e incluso una manera de vivir: una disposición para escuchar argumentos críticos, para buscar errores propios y para aprender de ellos" (Ibíd.). Lo cual se traducirá, más allá de un particular modo de proceder, en una idea respecto del mundo que el filósofo describe como tri-ismo o como la idea tripartita del mundo. Si quisiéramos representar lo anterior, podríamos recurrir a la polémica y fuertemente criticada pintura de Gustav Klimt grabada en la Universidad de Viena en 1900, la cual retrataba a la Filosofía, en La moldura de su Aula Magna, como un "grupo de figuras a la izquierda: génesis, experiencias fecundas, evanescencia. A la derecha: El Globo del Mundo, el Enigma del Mundo, abajo se eleva una figura luminosa, el saber" (Valverde 2000 459) que arrojará una teoría cosmológica de los tres mundos. 


\section{La teoría de los tres mundos como una propuesta abierta del mundo}

La TTM surge de una propuesta neodarwiniana o de mejora del darwinismo, la cual es analogizada al método epistemológico de «ensayo y error», largamente desarrollado por Popper en su teoría del conocimiento científico. Esta propuesta teórica se logra expresar a través de un esquema tetrádico, el cual describe la secuencia evolutiva de un organismo vivo del modo $P \rightarrow T S \rightarrow E E \rightarrow P$, "siendo " $P$ " un problema, "TS" la soluciones tentativas, "EE" la eliminación de errores" (Popper 2001c 225-226). En donde la vida de un organismo, a la luz de este algoritmo, se da como "una cuestión de resolución de problemas" (Popper 1994b 241) a través de propuestas tentativas frente a éstos.

Para Karl Popper, "vivir es solucionar problemas" (Popper 1988 99). Esto es, la vida de un organismo presupone problemas frente a los cuales éste hace intentos para solucionarlos. La vida porta un impulso estructural genético o innato, el cual se proyecta como un resolutor de problemas, haciendo de ello el camino que marca el vivir de un organismo biológico. Sin embargo, si reparamos en el vivir del ser humano, este organismo biológico nos "lleva -dice Popper-casi necesariamente a un programa de investigación que pide una explicación, en términos biológicos objetivos, de la emergencia de los estados de conciencia" (Popper 1994b 241). En efecto, Popper reparará en el hecho de que el ser humano al vivir o al ir enfrentando problemas del mundo, no solamente ofrecerá soluciones tentativas a éstos, sino que al mismo tiempo irá erigiendo estados de conciencia de ello. Pues bien, este será el problema del mundo que estimulará al pensador vienés, para adentrarse a un «programa metafísico -no falseable, pero si criticable- de investigación» que se dirigirá a lo mental o a esos estados emergentes de conciencia como un hecho del mundo.

\section{$\S 1$}

El primer paso popperiano en el estudio de esos estados de conciencia, en claves cosmológicas, será establecer un «giro biológico» de 
su filosofía, como es catalogado por W. W. Bartley en Philosophy of Biology versus Philosophy of Physics (1987). Planteando el hecho de que «siempre» fue un dualista cartesiano, ya que para él -Popper-era bastante obvio, aunque enigmático, la existencia de la mente o estados mentales en el mundo. Esta idea será postulada provisionalmente, desde el sentido común, aferrándose por ejemplo, al hecho de que existe un ideario colectivo que indica que "muchas cosas hablan, al parecer, a favor de la conjetura de que la idea de supervivencia después de la muerte entraña algún tipo de dualismo del cuerpo y la mente" (Popper 1980 173). Sin embargo, su dualismo no tendrá el mismo enfoque sustancialista propuesto por René Descartes.

Como es sabido, la distinción mente y cuerpo, que el filósofo francés postula en su Prima Philosophia, in Quibus Dei Existentia, \& Animae a Corpore Distinctio, Demonstratur de 1641, apunta a una distinción de tipo esencial respecto del cuerpo y el alma o mente respectivamente. En donde el primero responde a las veces de una res extensa y la segunda a una res cogitans, ambas sentencias son en repuesta a un ¿qué es el cuerpo? y ¿qué es el alma? Para Popper la pregunta ¿qué es? "¡no importa! [...] es Mejor preguntar: «¿Qué hace la mente?»" (Popper 1994b 251) y ¿qué hace el cuerpo? En efecto, el dualismo defendido por el pensador vienés, sólo será visto desde un dualismo de funciones que le conciernen únicamente a los «procesos o estados» tanto físicos como mentales. Siendo, por lo tanto, sólo un "casi cartesiano" (Popper 2001c 231 nota 43).

Para Popper, el estudio de lo mental, como realidad del mundo representa el "problema -cosmológico- más difícil y profundo de la filosofía" (Popper 1985 81), y al mismo tiempo desde el cual se ha levantado toda la filosofía occidental (cf. Popper 2001c 147). Así, el filósofo vienés entrará de lleno al problema cartesiano clásico del cuerpo y la mente mostrándose profundamente interesado por lo mental y por el problema mente-cuerpo, desde un plano más amplio como será el cosmológico.

La filosofía de la mente, de modo general, es vista como un estudio de la naturaleza y los procesos o estados mentales, situación que se 
da desde los orígenes de la reflexión filosófica. No obstante, para Popper gran parte de estos estudios se han mantenido en el ámbito de la creencia. Por ejemplo, Popper reconoce que las teorías antiguas "eran dualistas en la medida en que conferían al alma una condición muy especial y excepcional en el universo" (Popper 1980 180) con respecto al cuerpo. Por ejemplo, planteando intentos por resolver algunos aspectos de la relación del alma y el cuerpo, como es el caso de Aristóteles. Para el estagirita, cree Popper, "el desarrollo de las ideas éticas desempeñaban una función primordial en el desarrollo de la teoría del alma, la mente o el yo"(Popper 1980 180), como es el caso de la idea de supervivencia, reencarnación, superioridad, esencialidad o divinidad del alma o mente. Será Descartes quien vendrá a dar un "desplazamiento" (Id. 197) a esa forma de abordar lo mental, especialmente desde su obra Les Passions de L'Âme de 1649. Pues en ella se incluye no sólo la naturaleza de los fenómenos mentales como diferentes de los fenómenos físicos, sino su relación. Karl Popper se inclinará de manera primordial, en su estudio cosmológico de la mente, a la relación mente-cuerpo, desde la inquietud filosófica de "¿cómo es posible que los estados mentales -voliciones, sentimientos, expectativas- influyan o controlen los movimientos físicos de nuestras piernas? O, también ¿cómo es posible que los estados físicos de un organismo puedan influir sobre sus estados mentales?" (Popper 2001c 215). Es decir, se trata de poder explicar el interaccionismo o "influenciabilidad" (Popper 1988 104) causal entre la mente y el cuerpo, asimismo la diferencia entre los procesos y estados mentales y los físicos. Según John Searle, Karl Popper poco a poco irá mejorando a Descartes (cf. Searle 2006 62), revitalizando y contextualizando el dualismo a los avances científicos, pero por sobre todo adecuándolo al mundo.

La propuesta cosmológica popperiana puede ser descrita como «pluralista», en la cual se conjeturará la existencia, "at least" (Popper 1978 143), de un tri-ismo en la realidad. Ésta bosqueja, expresa Popper, una "picture of the universe" (Id. 166) de tres mundos. Propuesta popperiana que se erigirá adversativamente contra el monismo y el mero dualismo en lo referente a lo mental. Para Popper, esta mirada pluralista del universo, no representa una argumentación 
concluyente, aunque sí constituye una buena y no reduccionista proposición respecto del estudio del mundo, en particular de uno de esos mundos -el mental-. Por lo tanto, será este tri-ismo cosmológico desde el cual se articulará el pensamiento popperiano respecto de la mente, como un programa de investigación en el cual se develen los orígenes, características, hechos y procesos de lo mental en el mundo.

\section{$\S 2$}

"Cuando hablo con ustedes, no me dirijo a sus cuerpos, sino a sus mentes" (Popper 1997b 35). Esta sentencia popperiana, plantea la existencia de la mente, no como una ficción o un fantasma (Cf. Popper 1978 146), sino como algo que es y ante lo cual él y los otros seres humanos se enfrentan como una realidad del mundo. Del mismo modo, reconoce al cuerpo igualmente existiendo. Sin embargo, ante la dualidad cuerpo y mente que tiene enfrente, la centralidad está en esta última. Más aún si ésta, la mente, es "immensely important, especially from a human point of view" (Popper 1978 143). Por lo tanto, sin barrer con el cuerpo, Popper va a enfrentar el problema mente-cuerpo, situando su foco en la primera.

La idea cosmológica de mente, de cara al problema cuerpo-mente, se traduce en un mirar al cuerpo enlazado con lo mental o desde el punto de vista en el que "el cuerpo y el alma son en cierto modo inseparables" (Popper 2000 43). ¿Cómo es que se da esa relación? La tesis popperiana, que está en la base, es que "no podemos menos de admirar que la materia -cuerpo- pueda superarse a sí misma [...], produciendo mentes, propósitos y todo un mundo de productos de la mente humana" (Popper 1980 12). Es decir, a partir del cuerpo ha surgido la mente, esto es, que desde la materia se da lo que Popper llama, una «evolución emergente». Para defender esta emergencia o «fulguración», Popper desarrollará variadas críticas a la teoría de la identidad, al epifenomenalismo, el monismo psíquico o panpsiquismo y el monismo físico. Sin embargo, la crítica más interesante y más fructífera para exponer su pluralismo emergentista, será de frente a 
aquellos que postulan la no existencia de lo mental o la sola posibilidad de un monismo ${ }^{4}$ materialista o fisicalista.

En esta crítica, Karl Popper compartirá con el monismo fisicalista el paradigma cosmológico que significa la materialidad de la realidad para el estudio del universo, no obstante no coincidirá en la negación de lo mental, debido a que el fisicalismo sólo se ha obstinado en la idea de Thomas Hobbes. Éste postulaba que "el mundo [...] es corpóreo, es un cuerpo, $[. .$.$] cada parte del cuerpo es, a su vez cuerpo, [. .$.$] y$ lo que no es cuerpo no es parte del Universo. Y como el Universo lo es todo, lo que no forma parte de él es nada y, por consiguiente, no existe" (Hobbes 2003 553). A raíz de esta negativa se suprimirán los fenómenos mentales, lo cual se irá traduciendo en un mecanicismo del hombre que llegará a postular la idea del «hombre máquina», o irá convergiendo a un conductismo radical. Basicamente, hace "que los procesos conscientes y mentales no existen -por lo tanto, dice Popper-. su existencia se puede «repudiar»" (Popper 1980 59). Sin embargo, la postura fisicalista al ir rechazando lo mental, pasando por alto el sentido común o los experimentos ${ }^{5}$ que apelan a la existencia de la mente, obvian el hecho de que simplifican y clausuran, al mismo tiempo, el universo físico. La teoría fisicalista, al hegemonizar el universo sólo en lo material, cierra y obstruye en el universo toda creatividad, estrechando hacia un punto de vista reduccionista de éste. Por lo tanto, el monismo fisicalista se hace pobre y cerrado para explicar esa evolución creadora del universo, debido a que todo queda preso de la unidad física, la cual pese a los cambios sigue siendo unidad u ordenación física, suponiendo la idea de que "nada hay nuevo bajo el sol" (Popper 1980 15) o desconociendo que "la

\footnotetext{
4 Vale hacer notar que, en The Self and its Brain también se da una crítica al Monismo Metal o Pansiquismo, la cual arroja la idea de que este monismo es estrecho para explicar no sólo a la mente, sino también cómo ésta ha emergido. De ahí que esta teoría no ofrece una mejor comprensión respecto de lo mental, y con ello, comparte con el monismo material su simplicidad o estrechez explicativa.

5 Estos experimentos tienen que ver con aquellos estados de conciencia que afectan al cuerpo, como es el caso del sufrimiento o dolor humano. Karl Popper expone en The Self an its Brain (1977) el ejemplo del dolor de muela, el cual nos conduce materialmente al dentista, esto es, mueve físicamente el cuerpo.
} 
emergencia del hombre, [...] la creatividad del universo se ha hecho evidente"(Ibíd.), dice Popper. De esta forma, compartiendo con los fisicalistas la existencia de un mundo físico, sin embargo éste no es cerrado, sino que de él se genera otro mundo, el mundo de los estados mentales o de la mente, ampliando el monismo a un dualismo de mundos, esto es, mente y cuerpo. No basta con el fisicalismo, este es estrecho 0 «Poverty» ${ }^{6}$, puesto que su clausura explicativa respecto a una posibilidad de realización creativa o novedosa del universo físico, no contempla la creatividad del universo, y en ella, por ejemplo, la «emergence of mind», como postula Karl Popper en su primera conferencia pronunciada en el Darwin College de Cambridge en 1977.

Desde este universo abierto se genera un enfoque biológico de la realidad, en el que se ve, "con toda ingenuidad -dice Popper- a la mente humana como si fuera un órgano corporal altamente desarrollado" (Popper 1994b 254), el cual emerge evolutivamente del cuerpo como otra entidad del mundo, proyectando su quehacer a la conservación-como resolutor de problemas- del cuerpo, distanciándose de cualquier interpretación sustancialista o esencialista respecto de ella, y en segundo lugar conservando el pluralismo cosmológico. Por ello Popper sentencia: "no defiendo la idea de sustancia en ninguna de sus variantes, ni siquiera en el terreno de la física, y menos aún en el de la conciencia, en la psicología [...] el viejo problema del cuerpo y el alma iba ligado a la idea de sustancias. Pero la teoría de las sustancias se esfumó" (Popper 2000 120). Por lo tanto, negando la sustancialidad cosmológica, no sólo del cuerpo, sino también de la mente, abrirá a esta última hacia un acaecimiento cosmológico -nuevamente- novedoso o expansivo, en el que la mente será vista como un «sistema de control plástico» de nuestro organismo evolutivo, que interactúa con el cuerpo y viceversa, al modo de dos hechos o estados de un organismo biológico ${ }^{7}$ reales, los cuales se intervienen, conmutan e

6 El fisicalismo, en su afán de explicar el universo, es igualmente mísero que el historicismo en lo que respecta a su poder explicativo, como lo expresa Popper con el título de su obra The Poverty of Historicism.

7 Vale hacer notar, que al referirse a este organismo biológico, Karl Popper siempre está pensando en un organismo sano o en perfecto estado. 
interactúan entre sî́. Más aún, "Considero -dice Popper- útil y legítimo hablar de dos tipos de estados (o acontecimientos) -distintos- en interacción de carácter físico-químico y mental" (Popper 2001c 233). De esta forma, Popper abrirá el mundo físico, no sólo a una evolución creativa en él, sino también a la capacidad de ser influido por lo mental y viceversa. No obstante, "nuestra mente -dice Popper- [...] no es nunca una mera "corriente de la conciencia», una corriente de experiencia" (Popper 1980 144) que afecta al cuerpo, sino que en ella se promueven productos suyos, como son los "problems, arguments, reasons (and also works of art and many other things) [...] One of my central theses is that reason (or the validity of reasons), arguments, and even problems, can influence human behaviour and therefore the physical world" (Popper 1974 1073). De esta forma, los productos mentales o de la mente humana ${ }^{9}$ existen en el mundo. Por ejemplo, una teoría científica afecta no sólo al estado mental en su operación o corriente ${ }^{10}$ consciente, sino también al estado físico del organismo, lo que se traduce en que esos productos mentales existen y son reales, de acuerdo a la influencia causal que ejercen.

\section{$\S 3$}

Pues bien, más allá del mundo físico y del mundo mental, existe el mundo de los productos de la mente, o más allá del monismo y del mero dualismo existe un tri-ismo del universo. Si bien, el dualismo es pluralista desde un punto de vista monista, "yo, por mi parte -dice

8 Esta idea respecto de la mente puede ser relacionada con la de un relé, en el caso de un circuito eléctrico, ya que estos aparatos además de controlar una secuencia eléctrica también conmutan la energía que hay en ellos, esto es, transforman el contenido energético que hay en ellos. En efecto, lo que acontece con la mente no es el solo hecho de interactuar con el cuerpo y controlarlo, como aquel piloto del navío, sino que además conmuta el contenido que llega a ella.

9 Estos productos de la mente humana, asimismo pueden ser llevados a un estado físico al modo de ser "embodied or Physically realized". Así lo expone en su texto Three Worlds. p. 145.

10 Popper distingue en el conocimiento su aspecto «subjetivo», el cual se puede expresar como un «know» y su sentido «objetivo», esto es, el contenido de ese «know» o aquel conocimiento «sin sujeto cognoscente». 
Popper-, soy trialista. No dualista, sino trialista" (Popper 2000 43). Este tri-ismo se erigirá, plantea el pensador vienés, a partir de la idea de que "nuestra actual cosmología nos presenta como un hecho: un mundo en el que durante eones, no había ni rastro de vida o mente, en el que surgieron la vida, primero, y después la mente, e incluso un Mundo 3" (Popper 1980 225). De ahí que, conjetura el pensador vienés, "I will propose a view of the universe that recognizes at least three different but interacting sub-universes" (Popper 1978 143). Esta cosmología interaccionista se da en el sentido más amplio de la palabra (cf. Popper 2001c 148 nota3), es decir, en términos de que los mundos se interrelacionan y son además capaces de actuar e influirse unos con otros, en términos de un universo abierto, al menos, en su carácter tripartito.

Este tri-ismo interaccionista abierto, representa un prisma investigativo que no quiere diluir o restringir los problemas del mundo, sino abrirlos hacia un plurarismo interaccionista del universo. Que puede presentar más de tres mundos o que éstos presenten subdivisiones o sub-mundos (cf. Popper 1978 143) en ellos. Así, plantea Popper, "I do not pretend that I can refute these two views -dualism $y$ fisicalism-; but I can challenge them, by offering and defending a pluralist view" (Id. 148). De ahí que, "hemos de ser pluralistas, al menos de entrada" (Popper 2001c 267) respecto del universo, ya que de esta manera no se clausuran los problemas o dificultades filosóficas del mundo.

En este más allá del monismo y del dualismo, el pensador vienés postulará su cosmología como la TTM que contempla, como en repetidas veces nos lo hace ver en Three Worlds:

"There is the physical universe, world 1, with its most important sub-universe, that of the living organisms.

World 2, the world of conscious experience, emerge as an evolutionary product from the world of organisms.

World 3, the world of de products of the human mind, emerges as an evolutionary product from world 2"(1978 166167$)$. 
Los tres mundos se encuentran en un constante actuar o influenciabilidad de uno sobre otro, en un «feedback» o en un «dar y recibir», el cual tiene efectos causales reales sobre los otros mundos. De esta forma, cada uno de los mundos no se encuentra clausurado o cerrado a la repercusión real de los otros mundos, debido a que "como mínimo -se debe dar, plantea Popper-, la apertura causal de lo que voy a llamar el «Mundo1» hacia el «Mundo 2», y la apertura causal del «Mundo 2» hacia el «Mundo 3», y viceversa" (Popper 1986 136). En efecto, de un problema cosmológico dual se transita a un problema poniendo atención de manera especial, al Mundo 3 o tercer mundo. Ya que, por ejemplo, "el problema mente-cuerpo podría ser transformado por completo si llamamos en nuestra ayuda -cree Popper- a la teoría del mundo 3" (Popper 1994b 253) o de este tercer gran milagro (cf. Popper 1986 136) del mundo.

Este tercer ámbito es el real o del mundo, "más o menos tan reales como las mesas o las sillas físicas" (Popper 1994b 246). En donde, para el pensador vienés, algo "es real -o existe- si y sólo si, puede interactuar con los miembros del Mundo 1, con cuerpo físico [...] -o- algo existe, o es real, si y sólo si, puede recibir una patada y puede en principio, devolverla" (Popper 1986 138). Este carácter de realidad hace que estas entidades tercermundanas no sean ficciones o hipóstasis fuera de la existencia del mundo, sino que corresponden a objetos abstractos -versus los objetos concretos del Mundo 1-. Inclusive, estos productos de la mente humana pueden ser "embodied, or Physically realized, in one, or in many World 1 physical objects" (Popper 1978 145) los cuales, finalmente, "may have a causal effect upon us, upon our World 2 experiences, and further upon our World 1 brains, and thus upon material bodies"(Id. 150). Habrá que indicar, que la realidad de este tercer mundo será sacada a relucir reiteradamente, por Popper, a partir del efecto causal que ejerce en el cambio del Mundo 1, del Mundo 2 y asimismo del propio Mundo 3. De este modo, el Mundo 3 es "parcialmente autónomo" (Popper 1986 143), aunque es una creación real o «producción de la mente humana», puesto que instaura su propio dominio (cf. Popper 2001c 116) de consecuencias o problemas no previstos o no descubiertos por el hombre. "Los problemas que descubrimos emergen como 
consecuencia no intencionada de nuestros productos del mundo 3 , por lo tanto son sólo indirectamente productos de nuestra mente [...] aunque nosotros mismos iniciáramos la geometría y la aritmética (o la teoría de los números), los problemas y los teoremas pueden haber existido antes de que alguien los descubriera" (Popper 1997b $6667)$. En efecto, este dominio de autonomía planteará sus propios problemas, los cuales trascienden a su productor, tomando un perfil de "sobrehumano por cuanto que sus contenidos son objetos de pensamiento virtuales más bien que actuales y en el sentido de que tan sólo pueden convertirse en objetos actuales de pensamiento un número finito de los infinitos objetos virtuales" (Popper 2001c nota 8$)$.

La realidad y autonomía de este tercer mundo, lleva al pensador vienés a reconocer que "es obvio que lo que denomino "el tercer mundo" tiene mucho que ver con la teoría de las Formas o Ideas de Platón" (Popper 2001c 106) y al mismo tiempo es él [Platón] el "descubridor del tercer mundo" (Id. 120). Encargándose además, de hacer explícitos algunos de los continuadores de este tercer ámbito autónomo y objetivo, entre los que se cuenta a Georg Friedrich Hegel, con su «espíritu objetivo», Bernhard Bolzano con las «proposiciones en sí», y de manera especial a Gottlob Frege (1848-1925) con su "tercer dominio" (Frege 1972 122) objetivo del pensamiento. Así, Popper reconoce que la invención del Mundo 3 no es de él, sino que "lo único que quizás tenga de nuevo es el haberla puesto en relación con un evolucionismo" (Popper 2000 109) cosmológico de carácteristicas tercermundanas. Pues éste goza de un estatuto que permite un punto de vista objetivo, real y autónomo del mundo. Estableciendo "la existencia del conocimiento objetivo -Mundo 3- como producto objetivo y autónomo de la mente humana" (Popper 1997b 29 30). Esto es, dar razón de la existencia a una «mente objetiva»-sin sujeto cognocente-, producto de la mente -subjetiva- humana, la cual exhibe en sus objetos abstractos una naturaleza «objetiva» y «autónoma», formulada lingüísticamente (cf. Popper 1986 137) respecto de la realidad. Escapando de la idea de un mundo subjetivista, relativista o de la creencia. 


\section{Los alcances cognitivos de la Teoría de los Tres Mundos}

Como hemos visto más arriba la propuesta tercermundana de Karl Popper es esencial en su empeño filosófico cosmológico de su pensamiento. No obstante, es mayormente tardía, quedando algunos conceptos y algunas propuestas no completamente aclaradas o, como plantean Mario Bunge y Rubén Ardila, "a medio elaborar" (Bunge 1988 27). De ahí que exploraremos sus alcances en un horizonte crítico contemporáneo, especialmente respecto de la mente, en lo que se refiere al problema mente-cuerpo y su relación. Ahora sí, en el marco de las ciencias cognitivas.

El mapa actual cognitivista de la filosofía de la mente no se encuentra bien definido o claramente delimitado, ya que "el filósofo de la mente (como todo filósofo que se precie de tal) es un especialista en descubrir los límites de lo conceptual" (Rabossi 1995 17). No obstante, el enfoque de los problemas respecto de lo mental, mayormente, se dividirá en dualistas y monistas, aunque "ninguna de estas familias de doctrinas es homogénea" (Bunge 1988 25).

En nuestros días la filosofía de la mente se levanta como estudio de la naturaleza de los fenómenos y procesos mentales siendo parte del desarrollo filosófico desde sus inicios. Sin embargo, sólo a partir de la Modernidad se comienza a dar un estudio más sistemático de los estados mentales y de sus problemas, especialmente del problema mente-cuerpo. René Descartes será el punto de arranque clásico de esta área de estudio de segundo grado de la filosofía. Es decir, tras Descartes las estrategias teóricas para enfrentar a la mente serán influidas, a favor o en contra, a partir de lo propuesto por el filósofo francés. No obstante, más allá de su sistematización y de las dificultades por él abiertas, el estudio de la mente y sus problemáticas será una cuestión gravitante para el ser humano, ya que en ella se intenta, por ejemplo, responder, al «¿qué somos?». De esta forma el estudio sistemático respecto de la mente estará atravesado por ciertos presupuestos antropológicos de corte especulativo, inclusive religiosos, que marcarán su proceder, pero al mismo tiempo, y un poco contrarrestando lo anterior, se plantearán hipótesis científicas que buscan ganar objetividad. 
Como podemos ver, la filosofía de la mente es un ámbito de estudio que se encontrará cruzado por planteamientos tanto metafísicos y científicos, a raíz de que el objeto en cuestión toca al ser humano en su vida, haciendo de esta filosofía, como plantea John Searle, no sólo importante por el aspecto epistemológico que su estudio representa, sino por lo relevante para la vida humana.

En 1949 Gilbert Ryle presenta su obra The Concept of Mind, con la cual el estudio sistemático de la mente experimentará un cierto punto de inflexión en su desarrollo. Ryle cuestionará fuertemente el dualismo cartesiano debido a que en él se dan «category-mistakes» (cf. Ryle 1002 33), ya que al relacionar mente-cuerpo, de manera sustancial, se ligan expresiones de distintos campos epistemológicos. Lo notable en lo planteado por el filósofo inglés, es que este error cartesiano fomenta la idea de un espíritu sustancial en el ser humano o un «fantasma en la máquina»; dice Ryle, "I shall often speak of it -de la «doctrina oficial» como llama a la idea de Descartes-, with deliberate abusiveness, as 'the dogma of the Ghost in the Machine'. I hope to prove that it is entirely false, and false not in detail but in principle. It is not merely an assemblage of particular mistakes. It is one big mistake" (Ibíd.). Este equívoco ha llevado a que dos campos de investigación humana, como la ciencia y la filosofía, se encuentren aportando sus propias respuestas a diferentes preguntas acerca de lo mental. Hecho que para Ryle, recuerda Popper, era culpa de los filósofos (cf. Popper 1980 170). De esta forma Ryle, en una forma de hacerlas confluir -ciencia y filosofía-, traslada el estudio de la mente hacia un antimentalismo, dejando el carácter sustancial de ésta y pasando a ser estudiada como una disposición a la conducta ${ }^{11}$. De ahí que Ryle haya generado un escenario o un telón de fondo en lo que respecta a la filosofía de la mente contemporánea, lo cual se puede describir como un movimiento ambiguo, en mayor medida, entre un dualismo de propiedades y un intento por evitar o diluir ese dualismo al integrar los fenómenos mentales al mundo físico (cf. Bechtel 1991 109). Lo cual hace que la filosofía de la mente, en la

11 Habrá que advertir que este viraje en el estudio de la mente en la conducta, no hace de la mente la conducta, sino su disposición a ella. 
actualidad, más que un estudio de «la mente» propiamente tal, ella está "defined by a group of problems" (Kim 1998 7), los cuales refieren a lo mental y a los procesos mentales. En este escenario del estudio filosófico de la mente de la segunda mitad del siglo XX; Karl Popper, desde su TTM a la mente, propondrá una "nueva teoría de la mente y del ego" (Popper 1997b 164), la cual tendrá la forma de una teoría de la plena conciencia y del ego o de su yo, a partir de la complejidad de los estados de conciencia del hombre.

\section{$\S 1$}

Esta teoría del self ${ }^{2}$-o plena conciencia- postula el hecho de que el yo está anclado al Mundo 3, al modo de un objeto tercermundano lingüístico, el cual genera una visión del mismo ser humano en el espacio y el tiempo, algo así como un "espejo de la conciencia" (Popper 1980 120). Este evento se produce por medio de teorías objetivas, que igualmente son inquilinas del Mundo 3, al modo de una «mente autoconciente» o plena conciencia. Así, "the very idea of a self depends on World 3 theories, especially upon a theory of time which underlies the identity of the self, the self of yesterday, of today, and of tomorrow" (Popper 1978 167). En efecto, luego de que el cuerpo, o más precisamente el cerebro, se ha desarrollado evolutivamente hacia la emergencia de la mente, se dio paso a que a través de la formulación lingüística, como un producto exosomático, emerja la conciencia del yo, la cual va arrojando o produciendo teorías -tercermundanas- respecto del cuerpo, la mente y la relación entre ambos. De esta forma, el yo constantemente promueve visiones del ser humano por medio de las cuales va tomando conciencia.Por ejemplo, de dónde está el cuerpo, en qué está la mente y asimismo la relación entre ambos. "Sin embargo, sugiero que la conciencia humana del yo -agrega Popper-, trasciende todo pensamiento puramente biológico" (Popper 1980 162).

12 Es más preciso self, ya que el término en inglés hace mención a «uno mismo» o «sí mismo», más allá del pronombre personal / o yo en el idioma inglés o castellano, respectivamente. 
Pues bien, este yo no se agota en lo individual, sino más bien se abre a lo público, trascendiendo a la sociabilidad o a todos los demás seres humanos como espejos que reproducen un yo mental y público, el cual es formulado lingüísticamente. De ahí que, "en cuanto yo, en cuanto seres humanos -mente-cuerpo-, somos todos nosotros productos del Mundo 3 que, a su vez, es un producto de incontables mentes humanas" (Popper 1980 144).

Pues bien, a partir de ese yo cultural tercermundano, efecto de la historia de la humanidad, o de aquello que los antropólogos, plantea Popper, llaman cultura (cf. Popper 1978 166), se dará una teoría respecto de la relación mente-cuerpo, la cual se materializará a través de "las historias, los mitos explicativos, las herramientas, las teorías científicas (sean verdaderas o falsas), los problemas científicos, las instituciones sociales y las obras de arte" (Popper 1980 44). Desde ella se conjeturará una idea tercermundana respecto de la mente, y asimismo, una forma de aprender a ser un «selves» o yoes. Desde esta autoconciencia humana, que para Popper es el "mayor de los milagros" (Id. 144), comenzará el pensador vienés a dar luces de un cognitivismo acerca de los estados mentales, sin perder de vista ese yo, no obstante con una suerte de semejanza, como aquel «fantasma de la máquina» que atacaba Gilbert Ryle.

\section{$\S 2$}

La propuesta cognitivista popperiana se da en términos de un yo que interactúa abiertamente al modo de "una relación de acoplamiento de reacción recíproca" (Popper 1988 114), o sea por medio de «interrelationships and interactions» con ella. De esta forma, la mente consciente -Mundo 2- es también causalmente influenciada por ese yo -Mundo 3-, debido a que nuestras experiencias concientes o psicológicas también cambian de acuerdo a la teoría que entraña ese yo. Ya que, plantea Popper, En Sociedad abierta. Universo abierto:

"Es claro que el lenguaje y la escritura son producto del hombre y un producto de la psique humana. Es también claro que en tanto que leemos algo somos muy fuertemente dependientes 
de aquello que está allí. Nuestra vivencia al leer un libro -continúa el pensador vienés-guarda naturalmente una relación muy fuerte respecto del texto del libro. Y esto muestra que, en la forma más sencilla, si bien no es la más importante, la dependencia del mundo de las vivencias psicológicas del mundo 3 , esto es, del mundo de los productos de nuestro espíritu. Pero la más importante es que las consideraciones sobre el mundo 3 ofrecen una clave para explicar biológicamente el mundo 2" (1988 105, énfasis mío).

Del mismo modo, este yo interactúa con el cuerpo o, más precisamente, con el cerebro. Esta ligazón dualística -no epifenomenalistase da en términos de que "el yo, en cierto sentido, toca el cerebro del mismo modo que un pianista toca el piano o que un conductor acciona los mandos de un coche" (Popper 1980 557) ${ }^{13}$, por una suerte de necesidad disposicional del yo ${ }^{14}$ de relacionarse o interactuar con su cerebro. De ahí que, "el yo está, en parte por nuestras teorías del yo, conectado con su cuerpo" (Id. 536), en términos de un yo activo que repercute sobre el cerebro al modo de fluctuaciones neuronales. Sin embargo, todo ello se da gracias a la mediación del Mundo 2 o la mente, que codifica o conmuta la afección del yo sobre ella, para luego traspasarla al cerebro. Por lo tanto, de esa relación entre el yo y el cerebro, podemos obtener información respecto de lo mental y también respecto del hombre. Esto es, que somos, dice Popper y de manera obvia, "embodied selves or minds" (Popper 1974 148). El ser humano es una mente al modo de un «yo encarnado o in-corporado». Por lo tanto, somos un Mundo 3 «en» ${ }^{15}$ un Mundo

\footnotetext{
13 Popper comenta además, que esta idea de un espíritu de la máquina ya la pensaba desde 1950.

14 Popper describe esta disposición humana como genidentidad, esto es, la condición innata o genéticamente programada del hombre de dar con su yo. Más abajo veremos que la genidentidad se dará gracias al Mundo 3, el cual posibilitará, a través de sus objetos tercermundanos, que el hombre vaya descubriendo su identidad.

15 Este «en» tampoco implica una identificación de los productos mentales con el cerebro, sino una ligazón.
} 
1, sin embargo ese «en» hace ostensible a la mente, no desde lo que es, sino desde lo que produce, esto es, encarnar un «sí mismo».

Esta idea de «sí mismo» popperiano es quizás lo más osado de la TTM, ya que desde ese cognitivismo que pone su asiento en la función mediadora entre el yo y su cerebro Karl Popper tomará, en parte, la senda desplegada por René Descartes cuando postula la glándula pineal como el órgano que permite la interacción entre el cuerpo y el alma, la cual se aloja en el cerebro (cf. Descartes 2005 79). Si bien para Popper, Descartes era mecanicista por el hecho de que el hombre sólo era cuerpo y alma, como dos cosas o sustancias que interactuaban, sí le reconoce al pensador francés el haber establecido que "sólo en el cerebro puede haber interacción entre el Mundo 1 y el 2, y en este punto podemos decir-agrega Popper-que Descartes fue realmente un precursor. Aunque sea revolucionaria para la ciencia moderna, lo único que hacemos es retomar de un modo u otro la idea fundamental de Descartes de que el Mundo 1 (que para Descartes era el mundo mecanicista) está abierto, en el cerebro, al Mundo 2" (606).

El pensador vienés postulará el hecho de que la interacción se da en el centro del habla, el cual está ubicado en el hemisferio izquierdo del cerebro. Esta idea surge a raíz de que las interacciones entre el yo y el cerebro están asociadas a las funciones superiores del lenguaje, a raíz de que el propio Mundo 3, y por ende el yo anclado en él, es lingüístico. "Esto indica - plantea Popper-que la plena conciencia -el yo- interactúa en el centro del habla de nuestro cerebro" (Popper 1997b 188). Esta conjetura anatómica, como la llama Popper, parte del hecho de que el cerebro presenta dos mitades -izquierda y derecha-, las cuales están unidas por una comisura cerebral. En el caso de romperse esa unión cerebral en el ser humano, éste a simple vista no presentará problemas. Sin embargo, debido a que el hemisferio derecho controla el izquierdo y viceversa, el hombre, al no tener ese puente, no puede controlar sus órganos izquierdos con los derechos, esto es, "no es conciente de los movimientos que realizan su brazo y mano izquierdas. No se trata -advierte Popper- de una doble personalidad... -o yo-, es una personalidad completa, pero sólo es 
plenamente consciente de las señales que la mitad izquierda de su cerebro recibe de la mitad derecha de su cuerpo" (Popper 1997b 190). Lo que sucede es que las operaciones del lado izquierdo del cuerpo no pueden ser informadas al centro del habla, ubicado en el hemisferio izquierdo del cerebro, debido a que "es el hemisferio izquierdo quien llama nuestra atención -o la atención del yo- sobre un objeto" (Popper 1980 602), gracias al centro del habla. Si bien la mente no es el centro del habla, lo que sí hace notorio este ejemplo, según Popper, es que es ahí en donde se da un hecho productivo de ella que relaciona el «yo y su cerebro» como un «sí mismo». De ahí que, sentencia Popper en la primavera de 1969, en las Conferencias de Kenan de la Universidad de Emory, "what I have called 'world 2' -the world of the mind-becomes, on the human level, more and more the link between world 1 and world 3".

Como hemos podido apreciar, los alcances que la TTM tiene son amplios y profundos en el plano de las ciencias cognitivas. No obstante, la precisión respecto de éstos no es completa o definida, por el propio carácter problemático de la ciencia cognitiva y por otra parte porque la propuesta popperiana oscilará adversativamente entre elementos de carácter dualista interaccionista y materialista -eliminativista, emergentista y funcionalista. El primero de los enfoques, es particularmente el desplegado por el coautor de el $Y_{0} y$ su Cerebro, John Eccles. La mente es diferente del cerebro, ya que ésta ejerce una función superior a los procesos neuronales, aunque siempre interactuando con ellos, por medio de su interpretación y control. Si bien Eccles comparte el interaccionismo con Popper y también sus tres mundos, el primero pondrá su acento en los estados y procesos mentales, esto es, en el Mundo 2, en cambio Popper se inclina por los productos mentales o tercermundanos. Es el caso del «materialismo eliminativista», donde se cuentan autores como Paul y Patricia Churchland, quienes plantean la eliminación de los estados mentales, ya que estos sólo son un eco de la Folk Psychology -defendida por funcionalistas-. La teoría popperiana tendrá una fuerte crítica, sobre todo por su reduccionismo de la mente a los estados meramente neuronales. Sin embargo, compatirá con ellos el rescate de la utilización de los avances científicos, especialmen- 
te neurobiológicos, y también la esperanza de nuevos progresos neurocientíficos que resguarden el no caer en el psicologismo. En el caso del «emergentismo», en el que encontramos a John Searle y Mario Bunge, quienes se distancian del monismo y del dualismo sustancial, pero no así del materialismo psicobiológico, Popper compartirá con ellos su dualismo de propiedades, el cual consiste en que los procesos cerebrales o neurológicos, por su complejidad, hacen emerger propiedades diferentes a las cerebrales, Ilamadas propiedades mentales, las cuales se dan dentro de un proceso evolutivo. Por último, el «funcionalismo», con autores como Hilary Putnam y Jerry Fodor, quienes postulan que los procesos cerebrales son distintos de los procesos mentales, postulando estados funcionales del cerebro y del organismo, y asimismo abriéndose a un dualismo «cerebro-función cerebral», con el que Popper estará de acuerdo. No obstante, las discrepancias estarán en el materialismo que ostenta ese funcionalismo, especialmente en Putnam, al relacionar estados mentales «sólo» con sus estados funcionales o con el papel que juega la mente en la conducta.

Como podemos apreciar el desarrollo y alcance de la filosofía de la mente popperiana se enmarca en el plano adversativo con las filosofías de la mente contemporáneas, en términos de una "'nueva filosofía de la mente'" (1995, énfasis mío), como la llama Pascual Martínez. La cual no tiene una definición clara respecto del marco categorial que la rige. Haciendo que el poder explicativo e innovación teórica del enfoque popperiano, al problema mente y su autoconciencia, no sea concluyente respecto de sus alcances. He ahí que, como plantea Patricia Churchland, estamos a la espera de que Karl Popper, con su trabajo, traiga considerables noticias "not only in philosophy, but also in psychology and neuroscience" (Churchland 1981 99). Puesto que el filósofo vienés traspasa la línea filosófica objetivista que siempre había planteado hacia dimensiones insospechadas de su propia filosofía. En término de proyecciones investigativas que están lejos de ser dilucidadas, no sólo por lo tardío de ellas, sino por la importancia y el rol que ésta cumple en el pensamiento popperiano. Sólo nos atrevemos a reparar en un hecho biográfico que apunta a que el estudio acerca de la psique está de manera temprana en el pensamiento de 
Karl Popper ${ }^{16}$, quizás no siendo algo completamente excéntrico en su filosofía, sino una suerte de un filosofar consecuente con ese extraño racionalismo al que referiamos al comienzo de nuestro escrito.

\section{Conclusión}

La TTM nos abre hacia una dimensión del pensamiento popperiano que muchas veces es obviado como es su racionalismo crítico, no sólo en términos de una simple base especulativa para su formalismo, como lo demanda Mariano Artigas en su obra Karl Popper: Una Búsqueda sin Término de 1979, ni la mera ideología estructural que postula Miguel Quintanilla en su obra Idealismo y Filosofía de la Ciencia de 1972, ni el mero platonismo como lo ven tanto Mario Bunge en su obra Materialismo y Ciencia de 1981, como Anthony O'Hear en su texto Karl Popper de 1980, sino que en esta propuesta popperiana se da una filosofía radical, de carácter ilustrado, respecto de un mundo, incluido el hombre en ello. "Por racionalista entiendo un hombre que quiere comprender el mundo y aprender mediante la discución con otros" (Popper 1998 46). Esta actitud de razonalibidad se traduce en una modestia intelectual en términos de estar dispuesto a modificar las propias afirmaciones, admitiendo dudas y exigiendo el test en cada una de sus propuestas. Popper mismo dirá: "el principio de que todo está sujeto a crítica" (Popper 1994 677).

Como hemos visto, para la filosofía popperiana no hay autoridad ni dogmas, "cada fuente -tradición, razón, imaginación, observación, o lo que sea- es admisible y se puede utilizar" (Popper 2001 75). Lo que obliga a que todo el pensamiento del filósofo vienés se desarrolle en términos amplios como razones válidas o críticas contra sus propias afirmaciones y las de sus rivales. En donde se hace efectivo lo planteado en el Prefacio de la edición inglesa de Logik der Forschung, "hago equivalente la actitud racional y la actitud crítica"

16 Como un hecho positivo, el pensador vienés se doctoró en 1928 con la tesis Sobre la Cuestión del Método de la Psicología del Pensamiento, dirigida por el psicólogo Karl Bühler. 
(Popper 1986 17). Así la TTM con sus propuestas y alcances no es más la materialización de "la fe en la razón" (Popper 1994 677). Esto es, una teoría esperanzadora como el título de su autobiografía intelectual: una Búsqueda Sin Término. En donde la labor filosófica sigue una máxima en la que "tenemos el deber de seguir siendo optimista" (Popper 2001 156). Lo cual se traduce en que su TTM es una propuesta filosófica aún en desarrollo y su fertilidad, como un objeto tercermundano, está lejos de ser un evangelio de la verdad. Ella no busca totalizar o integrar el mundo, sino problematizarlo penetrando en sus misterios -por ejemplo la mente-, eso si, recordando que nunca se podrán exponer explicaciones últimas o plenamente satisfactorias respecto de ellos. Pese a ello, esta cosmología no anula el deseo de ir más lejos, ni favorece el empequeñecimiento de los problemas, sino a pensar en ellos como un reto filosófico de la razón.

Soy, dice Popper, "a tottering old metaphysician" (Popper 1974 993), y es precisamente ese tambalear en los enigmas del mundo el que se da en la TTM como una propuesta cosmológica, que irá oscilando en su desarrollo crítico respecto de la emergencia real de la mente, su interacción con el cuerpo y especialmente la posibilidad de un pluralismo cosmológico. En el que, por ejemplo, se constituye el yo humano. Representando ésta un rol relevante en la filosofía popperiana, no sólo por sus alcances cognitivistas, sino porque en ella se deja ver el sentido metafísico de la filosofía popperiana que puede, como lo reconoce el propio autor, ser un "plan perverso" (Popper 2001 27) para su epistemología, pues avanza como un rezagado tardío de la llustración que postula el lema Sapere aude el cual desafía a la filosofía popperiana a utilizar su propia racionalidad, para ver nuevos problemas, quizás donde nadie los ha visto, y encontrar nuevos modos de resolverlos.

\section{Bibliografía}

Artigas, Mariano. Karl Popper: Búsqueda Sin Término. Madrid: Magisterio Español, 1979.

Beakley, Brian; Ludlow, Peter (edits). The Philosophy of Mind: Classical Problems / Contemporary Issues. London: Massachusetts Institute of Technology, 4ª edición, 1997. 
Bechtel, William. Filosofía de la Mente. Una Panorámica para la Ciencia Cognitiva. Madrid: Tecnos, 1988.

Bochenski, I.M. La Filosofía Actual. México: Fondo de Cultura Económica, edición 2a, 1980.

Bühler, Karl. Psicología de la Forma (Cibernética y Vida). Madrid: Morata, 1965.

Bunge, Mario; Ardila, Rubén. Filosofía de la Psicología. Barcelona: Ariel, 1988.

Bunge, Mario. El Problema Mente-Cerebro. Un Enfoque Psicobiológico. Madrid: Tecnos, 2a edición, 1988.

Bunge, Mario. Materialismo y Ciencia. Barcelona: Ariel, 1981.

Bunge, Mario. Emergencia y Convergencia. Gedisa, Buenos Aires, 2004.

Campbell, Keith. Cuerpo y Mente. México: Universidad Autónoma de México, 1987.

Chalmers, David. La Mente Consciente. En Búsqueda de una Teoría Fundamental. Barcelona: Gedisa, 1999.

Churchland, Patricia. Is Determinism Self-refuring?. En: Mind, New Series, Vol 90, No. 357, Jan., 1981.

Churchland, Patricia. Neurophilosophy. Toward a Unified Science of The MindBrain. Cambridge: MIT, 2000.

Churchland, Paul. Materia y Conciencia. Introducción Contemporánea a la Filosofía de la Mente. Barcelona: Gedisa, 2ª edición, 1999.

Dennett, Daniel. Dulces Sueños. Obstáculos Filosóficos para una Ciencia de la Conciencia. Buenos Aires: Katz, 2006.

Descartes, René. El Tratado del Hombre. Madrid: Alianza, 1990.

Descartes, René. Las Pasiones del Alma. Buenos Aires: Edaf, 2005.

Descartes, René. Meditaciones Metafísicas con Objeciones y Respuestas. Madrid: Alfaguara, edición, 1977.

Eccles, John. How The Self Controls its Brain. Berlin Heidelberg: SpringerVerlag, 1994.

Eccles, John. The Human Mystery. The Gifford Lectures University of Edinburgh 1977-1978. London: Routledge \& Kegan Paul, 1984.

Fodor, Jerry. El Lomo y el Experto. El Reino de la Mente y su Semántica. Barcelona: Paidós, 1997. 
Fodor, Jerry. Psychological Explanation: An Introduction to the Philosophy of Psychology. New York: Ramdom House, 1968.

Frege, Gottlob. Lógica y Semántica. Valparaíso: Universidad Católica de Valparaíso, 1972.

Gardner, Howard. La Nueva Ciencia de la Mente. Paidós, Barcelona, 1987.

Goldman, Alvin. Epistemology and Cognition. Harvard University, 1986.

Guttenplan, Samuel (edit). A Companion to the Philosophy of Mind. Blackwell, 1996.

Houdé, Olivier et al. Diccionario de Ciencias Cognitivas. Neurociencia, Psicología, Inteligencia Artificial, Lingüistica y filosofía. Buenos Aires: Amorrortu, 2003.

Kenny, Anthony. La Metafísica de la Mente. Filosofía, Psicología, Lingüistica. Barcelona: Paidós, 2000.

Kim, Jaewon. Philosophy of Mind. Oxford: Westview Press, 1998.

Martínez, Pascual. La Nueva Filosofía de la Mente. Barcelona: Gedisa, 1995.

Mosterín, Jesús. Entrevista con Karl Popper, en Arbor: Ciencia, Pensamiento y Cultura, № 522, 1989.

O'Hear, Anthony. Karl Popper. London: Routledge \& Kegan Paul, 1980.

Popper, Karl. Búsqueda sin Término. Una Autobiografía Intelectual. Madrid: Tecnos, 3a edición, 1994b.

Popper, Karl. Conjeturas y Refutaciones. El Desarrollo del Conocimiento Científico. Barcelona: Paidós, $2001 \mathrm{~b}$.

Popper, Karl. Conocimiento Objetivo. Un Enfoque Evolucionista. Madrid: Tecnos, 4a edición, 2001c.

Popper, Karl. El Cuerpo y la Mente. Escritos Inéditos Acerca del Problema CuerpoMente. Barcelona: Paidós, 1997b.

Popper, Karl. El Mito del Marco Común. En Defensa de la Ciencia y la Racionalidad. Barcelona: Paidós, 1997a.

Popper, Karl. El Porvenir está Abierto. Barcelona: Tusquets, 3a edición, 2000.

Popper, Karl. El Universo Abierto. Argumento a Favor del Indeterminismo. Post Scriptum a la Lógica de la Investigación Científica. Vol II. Madrid: Tecnos, 1986.

Popper, Karl. El Yo y su Cerebro. Barcelona: Labor, 1980. 
Popper, Karl. La Lógica de la Investigación Científica. Madrid: Tecnos, $2^{\mathrm{a}}$ edición, 2001 a.

Popper, Karl. La Responsabilidad de Vivir. Escritos sobre Política, Historia y Conocimiento. Madrid: Paidós, 1995.

Popper, Karl. La Selección Natural y el Surgimiento de la Mente. En. Olivé, León; Martínez, Sergio (comp.), Epistemología Evolucionista. Barcelona: Paidós, 1997.

Popper, Karl. La Sociedad Abierta y sus Enemigos. Barcelona: Paidós, 1994a.

Popper, Karl. Los Dos Problemas Fundamentales de la Epistemología. Basado en Manuscritos de los años 1930-1933. Madrid: Tecnos, 1998a.

Popper, Karl. Realismo y el Objetivo de la Ciencia. Post Scriptum a la Lógica de la Investigación Científica. Vol. I. Traducción castellana: Madrid: Tecnos, $2^{\text {a }}$ edición, 1998b.

Popper, Karl. Replies to my Critics (1974). Edición inglesa: Schilpp, Paul Artur (comp). The Philosophy of Karl Popper, La Salle (Illinois), Open Court, 1974, Vol. 1, Vol. 2.

Popper, Karl. Sociedad Abierta, Universo Abierto. Conversación con Franz Kreuzer. Madrid: Tecnos, 2ª edición, 1988.

Popper, Karl. Teoría Cuántica y el Cisma de la física. Post Scriptum a la Lógica de la Investigación Científica. Vol. III. Madrid: Tecnos, 1985.

Popper, Karl. Three Worlds (1978). The Tanner lecture on Human Values. Delivered at The University of Michigan, April 7, 1978.

Popper, Karl. Un Mundo de Propensiones. Madrid: Tecnos, 2a edición, 1996.

Putnam, Hilary. La Trenza de Tres Cabos. La Mente, el Cuerpo y el Mundo. Madrid: Siglo XXI, 2001.

Putnam, Hilary. Representación y Realidad. Un Balance Crítico del Funcionalismo. Barcelona: Gedisa, 1990.

Queraltó, Ramón. Karl Popper. De la Epistemología a la Metafísica. Sevilla: Universidad de Sevilla, 1996.

Rabossi, Eduardo (comp). Filosofía de la Mente y Ciencia Cognitiva. Barcelona: Paidós, 1995.

Rabossi, Eduardo (comp). La Mente y sus Problemas. Temas actuales de Filosofía de la Psicología. Buenos Aires: Catálogos, 2004.

Ryle, Gilbert. The Concept of Mind. Chicago: University of Chicago, 1984. 
Searle, John. La Mente. Una Breve Introducción. Bogotá: Norma, 2006

Searle, John. Mente, Lenguaje y Sociedad. Madrid: Alianza, 2001.

Searle, John. The Rediscovery of the Mind. Cambridge: MIT, 1992.

Valverde, José María. Historia de las Mentalidades. Vol. IV. Madrid: Trotta, 2000.

Wilson, Robert; Keil, Frank (edit). Enciclopedia MIT de Ciencias Cognitivas. Madrid: Síntesis, 2002. 